\title{
SER Performance Analysis of DF Cooperative System over Rayleigh Fading Channels
}

\author{
Ting Xia, Weiyao Xu \\ College of Information Engineering, Zaozhuang University, Zaozhuang 277160, China. \\ 412447672@qq.com
}

Keywords: SER, DF Cooperative System, Rayleigh Fading

\begin{abstract}
The symbol error rate (SER) performance of decode-and-forward (DF) relay cooperative system over flat Rayleigh fading channels is analyzed in this paper. Using the moment generating function (MGF) based analysis approach, the closed-form expressions for the average SER of coherently detected M-ary phase-shift keying (MPSK) for the system are derived. In addition, the SER performance of system with no cooperation is also given to compare with that with relay cooperation. Numerical results show that better SER performance can be achieved by increasing the average receive signal-to-noise ratio (SNR), and the SER performance of system with DF cooperation is better than that with no cooperation. The correctness of theoretical analysis is verified by simulation results.
\end{abstract}

\section{Introduction}

In the conventional point-to-point wireless communication, receiver cannot receive the messages if the channel or link is interrupted or disabled, which makes it impossible to guarantee the continuous communication. To overcome this weakness, cooperative communication has been created and applied widely in wireless networks [1]-[8]. It is developed based on the idea that all mobile users or nodes in a wireless network can send messages through mutual cooperation [1] and obtain the cooperative diversity gain [2]. Reference [3] put forth several cooperative communication protocols for wireless network. When a user assists other users to forward messages, the user will decode the messages received as a relay prior to forwarding, or simply amplify and then forward the messages. The former is called decode-and-forward (DF) protocol, while the latter is called amplify-and-forward (AF) protocol. Reference [4] introduced a cooperation strategy based on channel code, that is, coded cooperation (CC). Studies show that the cooperative communication techniques can improve not only the coverage and throughput of system, but also the quality of system transmission.

Reference [5] analyzed the average performance of AF relay cooperative system's symbol error rate (SER) over the Rayleigh fading channels with channel estimation error. The performance of DF cooperative system over Nakagami-m fading channels was studied in References [6] and [7]. Reference [8] illustrated the SER expression of the cooperative system using the DF protocol over the Rayleigh fading channels, but did not present the closed form, which resulted in the complicated algorithm. By carefully analyzing the SER performance of DF cooperative communication system, this paper derives the closed-form expressions for the average SER of the system modulated by using the M-ary phase-shift keying (MPSK) over the Rayleigh fading channels. The algorithm used in this process is very simple. In the end, the numerical calculation and computer simulation are carried out.

\section{System Model}

In the model of cooperative communication system with single relay as shown in Fig. 1, the relay $R$ adopts the DF protocol to process the signals received. There are two time slots in the 
communication process from the source node $S$ to the destination node $D^{[10]}$. Within the first time slot, $S$ sends the message $x$ to $D$ and $R$, and the average power of $x$ is $P_{1}$. Thus, $R$ and $D$ receive the following messages respectively:

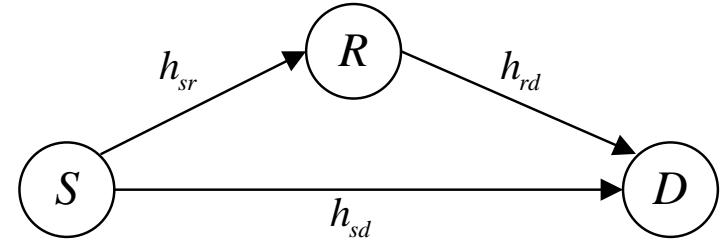

FIGURE 1. Cooperative System Model with Single Relay

$$
\begin{aligned}
& r_{s r}=\sqrt{P_{1}} h_{s r} x+n_{s r} \\
& r_{s d}=\sqrt{P_{1}} h_{s d} x+n_{s d}
\end{aligned}
$$

In which, $h_{s r}$ and $h_{s d}$ stand for the channel fading coefficients from $S$ to $R$ and $D$ respectively. It is assumed that every channel of the system is a flat Rayleigh fading channel; $h_{s r}$ and $h_{s d}$ are complex Gaussian random variables with the average value 0 and the variance $1 ; n_{s r}$ and $n_{s d}$ denote the additive white Gaussian noises (AWGN) over the links $S-R$ and $S$ - $D$ respectively, which are subject to the complex Gaussian distribution with the average value 0 and the variance $N_{0}$. Within the second time slot, the relay $R$ decodes the signal received, and then forwards the decoded signal to $D$ under the average power $\tilde{P}_{2}$. If the relay $R$ decodes accurately, there is $\tilde{P}_{2}=P_{2}$. Otherwise, the relay $R$ remains idle, so there is $\tilde{P}_{2}=0$. Therefore, the signal received by the destination node $D$ within the second time slot is:

$$
r_{r d}=\sqrt{\tilde{P}_{2}} h_{r d} x+n_{r d}
$$

In which, $h_{r d}$ is the channel fading coefficient over the $R-D$ link, which is a complex Gaussian random variable with the average value 0 and the variance $1 ; n_{r d}$ stands for the AWGN over the $R$ $D$ link, which is subject to the complex Gaussian distribution with the average value 0 and the variance $N_{0}$.

When the $S-R$ and $S-D$ links and $R$ accurately decode the signal received, their transient signalto-noise ratios (SNR) at the receiving end of the $R-D$ link are as follows:

$$
\begin{aligned}
& \gamma_{s r}=\left|h_{s r}\right|^{2} P_{1} / N_{0} \\
& \gamma_{s d}=\left|h_{s d}\right|^{2} P_{1} / N_{0} \\
& \gamma_{r d}=\left|h_{r d}\right|^{2} P_{2} / N_{0}
\end{aligned}
$$

In the DF relay cooperative system, it is assumed that the destination $D$ employs the maximal ratio combining (MRC) to combine the signals received within two time slots. Eventually, the total transient output SNR at the receiving end of the node $D$ is presented as follows:

$$
\gamma_{D}=\frac{\left|h_{s d}\right|^{2} P_{1}+\left|h_{r d}\right|^{2} \tilde{P}_{2}}{N_{0}}
$$

\section{MPSK Performance Analysis}

The conditional SER of coherently detected MPSK signals is obtained as follows ${ }^{[9]}$ :

$$
P_{M P S K}(\gamma)=\frac{1}{\pi} \int_{0}^{(M-1) \pi / M} \exp \left(-\frac{g_{M P S K}}{\sin ^{2} \theta} \gamma\right) d \theta
$$

In which, $g_{\text {MPSK }}=\sin ^{2}(\pi / M) ; \gamma$ stands for SNR. According to Reference [8], the source node $S$ sends the MPSK symbol, so the probability of the relay $R$ decoding erroneously is $P_{M P S K}\left(\gamma_{s r}\right)$, and there is $\tilde{P}_{2}=0$; the probability of the relay $R$ decoding accurately is $1-P_{M P S K}\left(\gamma_{s r}\right)$, and there is 
$\tilde{P}_{2}=P_{2}$, so we can obtain the conditional SER of the DF cooperative system as follows:

$$
P_{e}\left(\gamma_{D}, \gamma_{s r}\right)=\left.P_{M P S K}\left(\gamma_{D}\right)\right|_{\tilde{P}_{2}=0} P_{M P S K}\left(\gamma_{s r}\right)+\left.P_{M P S K}\left(\gamma_{D}\right)\right|_{\tilde{P}_{2}=P_{2}}\left[1-P_{M P S K}\left(\gamma_{s r}\right)\right]
$$

In which, the first item on the right of the equation denotes the conditional SER of the node $D$ when $R$ decodes erroneously, and the second item stands for the conditional SER of $D$ when $R$ decodes accurately. Equations (4) and (7) are substituted into Equation (9), so the conditional SER in Equation (9) can be presented as follows:

$$
\begin{gathered}
P_{e}\left(h_{s d}, h_{s r}, h_{r d}\right)=P_{M P S K}\left(\frac{\left|h_{s d}\right|^{2} P_{1}}{N_{0}}\right) P_{M P S K}\left(\frac{\left|h_{s r}\right|^{2} P_{1}}{N_{0}}\right)+P_{M P S K}\left(\frac{\left|h_{s d}\right|^{2} P_{1}+\left|h_{r d}\right|^{2} P_{2}}{N_{0}}\right) \times \\
{\left[1-P_{M P S K}\left(\frac{\left|h_{s r}\right|^{2} P_{1}}{N_{0}}\right)\right]}
\end{gathered}
$$

The channel fading coefficients are used to statistically average the conditional SERs in Equation (10). With Equations (8) and (10) as well as Equations (2.8) and (9.15) in Reference [9], the moment generating function (MGF) can help obtain the expression for the average SER of the DF cooperative communication system modulated by MPSK as follows:

$$
\begin{array}{r}
P_{e, M P S K}=\left(\frac{1}{\pi} \int_{0}^{(M-1) \pi / M}\left(1+\frac{g_{M P S K} P_{1}}{N_{0} \sin ^{2} \theta}\right)^{-1} d \theta\right)^{2}+ \\
\frac{1}{\pi} \int_{0}^{(M-1) \pi / M}\left(1+\frac{g_{M P S K} P_{1}}{N_{0} \sin ^{2} \theta}\right)^{-1}\left(1+\frac{g_{M P S K} P_{2}}{N_{0} \sin ^{2} \theta}\right)^{-1} d \theta \times\left(1-\frac{1}{\pi} \int_{0}^{(M-1) \pi / M}\left(1+\frac{g_{M P S K} P_{1}}{N_{0} \sin ^{2} \theta}\right)^{-1} d \theta\right)
\end{array}
$$

Assuming that the total transmission power of the system is $P_{T}=P_{1}+P_{2}$, this paper employs the power allocation $P_{1}=P_{2}=P_{T} / 2$. By letting $\bar{\gamma}=P_{T} / N_{0}$, and utilizing Equation (5A.24) in Reference [9], the closed-form expression for the average SER $P_{e, M P S K}$ in Equation (11) is derived as follows:

$$
\begin{gathered}
P_{e, M P S K}=\left(\frac{1}{\pi} \int_{0}^{(M-1) \pi / M} \frac{\sin ^{2} \theta}{\sin ^{2} \theta+g_{M P S K} \bar{\gamma} / 2} d \theta\right)^{2}+\frac{1}{\pi} \int_{0}^{(M-1) \pi / M}\left(\frac{\sin ^{2} \theta}{\sin ^{2} \theta+g_{M P S K} \bar{\gamma} / 2}\right)^{2} d \theta \times \\
\left(1-\frac{1}{\pi} \int_{0}^{(M-1) \pi / M} \frac{\sin ^{2} \theta}{\sin ^{2} \theta+g_{M P S K} \bar{\gamma} / 2} d \theta\right)=\left[I\left(1, \frac{g_{M P S K} \bar{\gamma}}{2}, \frac{(M-1) \pi}{M}\right)\right]^{2}+I\left(2, \frac{g_{M P S K} \bar{\gamma}}{2}, \frac{(M-1) \pi}{M}\right) \times \\
{\left[1-I\left(1, \frac{g_{M P S K} \bar{\gamma}}{2}, \frac{(M-1) \pi}{M}\right)\right]}
\end{gathered}
$$

In the above Equation, the function $I(\cdot, \cdot, \cdot)$ is defined as follows ${ }^{[9]}$ :

$$
\begin{gathered}
I(z, d, \theta)=\frac{1}{\pi} \int_{0}^{\theta}\left[\sin ^{2} \varphi /\left(\sin ^{2} \varphi+d\right)\right]^{z} d \varphi=\frac{\theta}{\pi}-\frac{\beta}{\pi}\left\{\left(\frac{\pi}{2}+\tan ^{-1} \alpha\right) \sum_{r=0}^{z-1}\left(\begin{array}{c}
2 r \\
r
\end{array}\right) \frac{1}{[4(1+d)]^{r}}+\right. \\
\left.\sin \left(\tan ^{-1} \alpha\right) \sum_{r=1}^{z-1} \sum_{l=1}^{m} \frac{T_{l r}}{(1+d)^{r}} \times\left[\cos \left(\tan ^{-1} \alpha\right)\right]^{2(r-l)+1}\right\}
\end{gathered}
$$

In which, $T_{l r}=\left(\begin{array}{c}2 r \\ r\end{array}\right) /\left[\left(\begin{array}{c}2(r-l) \\ r-l\end{array}\right) 4^{l}[2(r-l)+1]\right] ; \beta \triangleq \sqrt{\frac{d}{1+d}} \operatorname{sgn} \theta ; \alpha \underline{\underline{\Delta}}-\beta \cot \theta ;-\pi \leq \theta \leq \pi$. Equation (12) is the closed-form expression for the average SER performance of the DF relay cooperative system modulated by MPSK over the flat Rayleigh fading channels..

\section{Numerical Calculation and Simulation Results}

To verify whether the results of the above theoretical analysis are correct, the quaternary phase-shift keying (QPSK) and 8PSK modulated signals are used in the computer simulation and 
numerical calculation respectively to obtain the results presented in Fig. 1 and 2. Fig. 1 and Fig. 2 show the SER performance of DF cooperative system modulated by QPSK and 8PSK over the flat Rayleigh fading channels respectively, which is compared with the system performance during transmission without the relay. As revealed in such two figures, the accurate SER theoretical calculation obtains the results very close to the simulation results of the DF cooperative communication system, proving the correctness of the theoretical analysis in the above section. As we can see in the figures, the system performance with the relay cooperative transmission is better than that without the relay transmission.

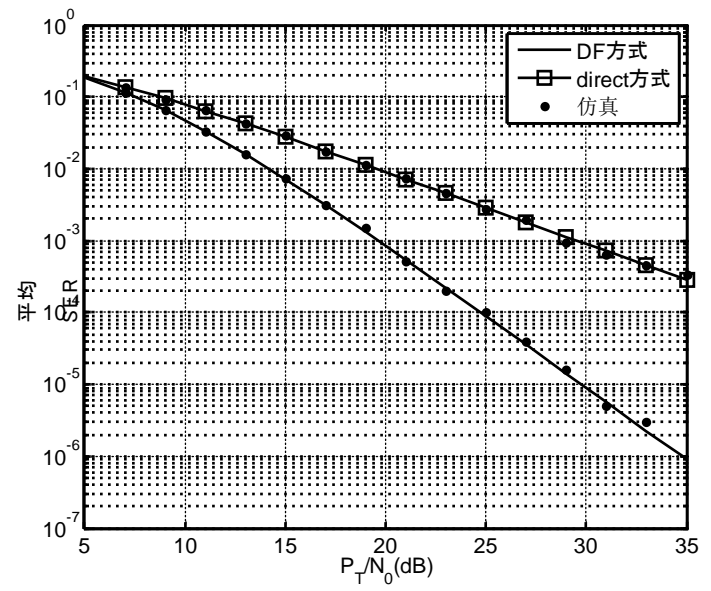

FIGURE 2. Average SER of DF Cooperative System Modulated by QPSK

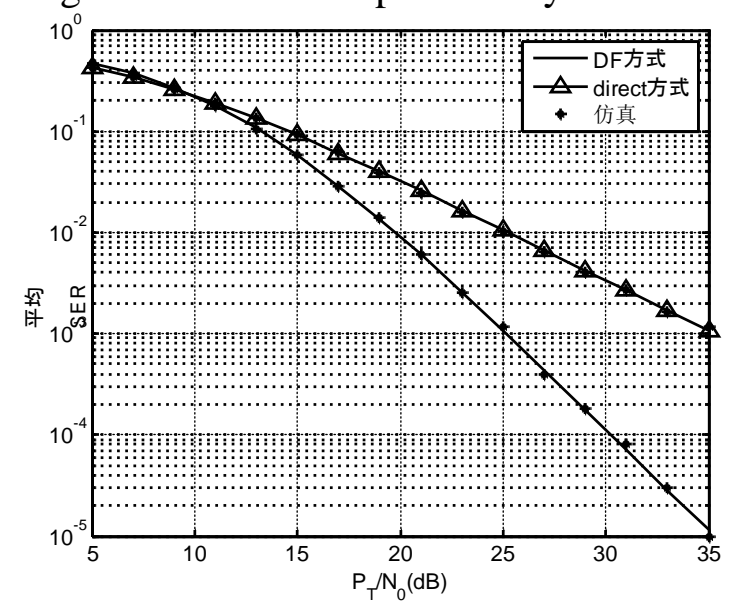

FIGURE 3. Average SER of DF Cooperative System Modulated by 8PSK

Additionally, it is also revealed in these figures that the gradual increase of the average SNR at the receiving end keeps improving the SER performance of DF relay cooperative system like the system without the relay transmission.

\section{Conclusion}

This paper analyzes the SER performance of the DF cooperative communication system over the flat Rayleigh fading channels, and employs the MGF technique to derive the accurate closed-form expression for the average SER of the system modulated by MPSK. This closed-form expression is so simple that the time-consuming numerical integration or computer simulation is abandoned. The simulation results prove the correctness of the theoretical analysis results. As shown in the results, the SER performance of the DF relay cooperative system is better than the system without the relay, and improving along with the gradual increase of SNR at the receiving end.

\section{References}

[1] Sendonaris A, Erkip E, Aazhang B. Increasing uplink capacity via user cooperation diversity. IEEE International Symposium on Information Theory, Cambridge, MA: 1998: 156. 
[2] Sendonaris A, Erkip E, Aazhang B. User cooperation diversity-Part I: system descrip-tion. IEEE Trans. on Communications, 2003, 51 (11): 1927-1938.

[3] Laneman J N, Tse D N C, Wornell G W. Cooperative diversity in wireless networks: efficient protocols and outage behavior. IEEE Trans. on Information Theory, 2004, 50(12): 3062-3080.

[4] Janani M, Hedayat A, Hunter T E, et al. Coded cooperation in wireless communi- cations: space-time transmission and iterative decoding. IEEE Trans. on Signal Processing, 2004, 52(2): 362-371.

[5] Wu Y, Patzold M. Performance analysis of amplify-and-forward cooperative communi- cation systems with channel estimation errors. 11th IEEE Singapore International Conference on Communication Systems, Guangzhou: 2008: 1620-1624.

[6] Ikki S, Ahmed M H. Performance of decode-and-forward cooperative diversity networks over Nakagami-m fading channels. IEEE Global Telecommunications Con- ference, Washington, DC: 2007: 4328-4333.

[7] Lee Y, Tsai M H. Performance of decode -and-forward cooperative communications over Nakagami-m fading channels. IEEE Trans. On Vehicular Technology, 2009, 58(3): 1218-1228.

[8] Su W, Sadek A K, Liu J K. SER performance analysis and optimum power allocation for decode-and-forward cooperation protocol in wireless networks. IEEE Wireless Communications and Networking Conference, 2005: 984-989.

[9] Simon M K, Alouini M S. Digital Communication over Fading Channels (2nd ed). New York: John Wiley \& Sons Inc, 2005.

[10] Liu K J R, Sadek A K, Su W, et al. Cooperative Communications and Net- working. New York: Cambridge, 2009. 